\title{
Relationship between diabetes mellitus type 1 and male reproductive function
}

\section{Sandro La Vignera, Rosita A. Condorelli, Aldo E. Calogero}

Department of Clinical and Experimental Medicine, University of Catania, Catania, Italy

\section{Introduction}

Diabetes mellitus type 1 (DM1) affects an increasing number of young men in reproductive age. Its prevalence increases at a rate of $\sim 3 \%$ per annum. DM1 may affect male reproductive function by acting on the hypothalamic-pituitary-testicular axis, causing sexual dysfunction and disrupting male accessory gland function. A recent study shows that men with DM1 have a smaller number of live births than controls. Little is known about sperm parameters and other aspects of the male reproductive function in these patients.

Aim: to evaluate both conventional and non-conventional sperm parameters, serum gonadal hormones and didymo-epididymal ultrasound features in patients with DM1.

\section{Methods}

We enrolled:

- 30 patients with DM1 (aged 1835 years)

- 20 age-matched fertile healthy men and classified according to disease duration $(<5,5-10,>10$ years)

Exclusion criteria: patients with diabetic neuropathy, other endocrine disorders or conditions known to alter sperm parameters

The following parameters were assessed:

- conventional sperm parameters (WHO 2010 criteria);

- non conventional sperm parameters (mitochondrial membrane potential, degree of viability and/or apoptosis, sperm DNA fragmentation) by flow cytometry;

- serum total testosterone, $17 \beta-$ estradiol, LH, FSH and PRL

- testicular and epididymal morphometry by ultrasound scan before and after ejaculation.

\section{Results}

- DM1 patients had a significantly lower percentage of sperm progressive motility than controls: this abnormality was significantly lower in DM1 patients with long ( $>10$ years) than short ( $<5$ years) disease duration

- The alterations on non-conventional sperm parameters are shown in Figure $1 \mathrm{~A}$ and $1 \mathrm{~B}$

- Patients with DM1 compared to controls and those with long disease duration had a significantly higher cephalic and caudal epididymal diameters after ejaculation

- All the other parameters did not show any statistically significant difference

Fig 1. Non conventional sperm parameters in patients with DM1 and controls $(A)$ and classified according to disease duration $(B)$

$\mathbf{A}$

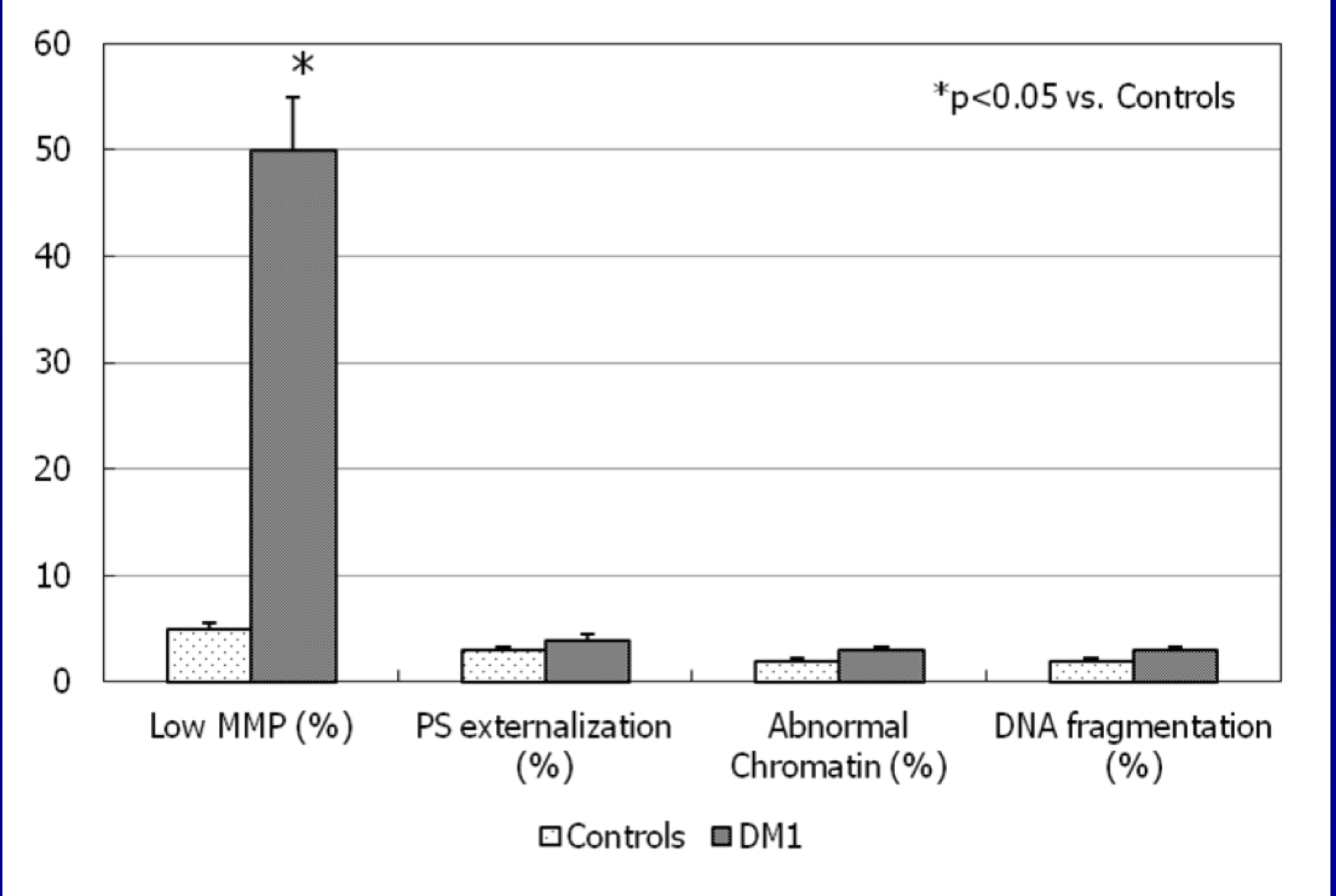

B

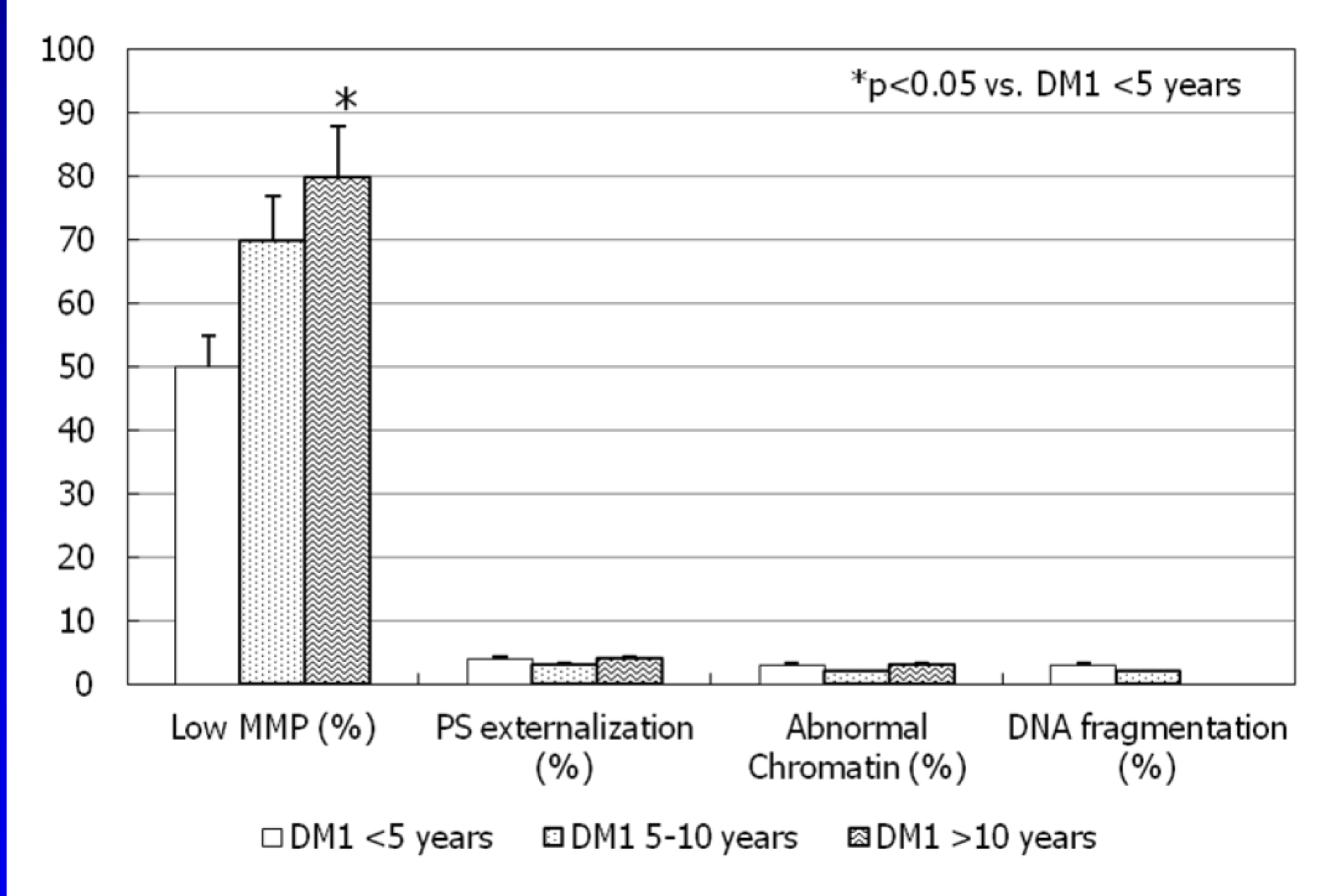

\section{Conclusions}

In conclusion, patients with DM1 had lower sperm progressive motility because of impaired mitochondrial function and epididymal post-ejaculatory dysfunction which cannot be ascribed to endocrinopathy and/or neuropathy. These findings may explain some fertility disorders in DM1 patients. 